Tropical Journal of Pharmaceutical Research December 2020; 19 (12): 2537-2543

ISSN: $1596-5996$ (print); 1596-9827 (electronic)

(C) Pharmacotherapy Group, Faculty of Pharmacy, University of Benin, Benin City, 300001 Nigeria.

\title{
Upregulation of miR-195a inhibits brain-derived neurotrophic factor in mouse hippocampus and may contribute to depression-like behaviors induced by chronic social stress
}

\author{
Xuping Wen ${ }^{1}$, Mingshuan Lin ${ }^{2 *}$ \\ ${ }^{1}$ Department of Student Affairs, Huzhou University Qiuzhen College, Huzhou, Zhejiang Province 313000, ${ }^{2}$ Department of \\ Clinical Psychological Psychiatry, Affiliated Haikou Hospital of Xiangya Medical College, Central South University, Haikou City, \\ Hainan Province 570208, China \\ *For correspondence: Email: shuanlm666@163.com; Tel: +86-0898-66151001
}

Sent for review: 8 September 2020

Revised accepted: 22 November 2020

\begin{abstract}
Purpose: To explore the effect of miR-195a on nerve cells in the hippocampal region of depressionmodel mice.

Methods: A chronic social defeat stress (CSDS) model was used as a depressed mouse model. In vivo, C57BL/6J mice received CSDS treatment or miR-195a antagomir. Depression-like behaviors were evaluated. In vitro, the target relationship between miR-195a and brain-derived neurotrophic factor (BDNF) was validated by luciferase reporter assays in HEK-293 cells. In primary cortical neurons, expression levels of miR-195a and BDNF mRNA were evaluated using quantitative polymerase chain reaction ( $q P C R)$. BDNF protein expression was determined by western blotting.

Results: The sucrose preference ratio and social contact of the CSDS group were significantly decreased, whereas the immobility time was significantly increased, compared with the control group ( $p$ $<0.05)$. Interestingly, the expression of miR-195a was upregulated in the CSDS group compared with control group $(p<0.05)$. Bioinformatics prediction and luciferase reporter assay data indicate that miR195a bound the BDNF 3' untranslated region. BDNF protein expression levels were significantly reduced by miR-195a mimic but increased by miR-195a inhibitor, compared with the negative control mimic group $(p<0.05)$. In vivo, miR-195a antagomir alleviated depression-like behaviors compared with CSDS group. In addition, miR-195a antagomir restored the expression of BDNF in mouse hippocampus in the CSDS group $(p<0.05)$.

Conclusion: MiR-195a inhibitor ameliorates depression-like behaviors of depressed mice by downregulation of BDNF, whereas upregulation of miR-195a inhibits BDNF expression in mouse hippocampus and may contribute to depression.
\end{abstract}

Keywords: Chronic social defeat stress, Depression, MiR-195, brain-derived neurotrophic factor, BDNF

This is an Open Access article that uses a funding model which does not charge readers or their institutions for access and distributed under the terms of the Creative Commons Attribution License (http://creativecommons.org/licenses/by/4.0) and the Budapest Open Access Initiative (http://www.budapestopenaccessinitiative.org/read), which permit unrestricted use, distribution, and reproduction in any medium, provided the original work is properly credited.

Tropical Journal of Pharmaceutical Research is indexed by Science Citation Index (SciSearch), Scopus, International Pharmaceutical Abstract, Chemical Abstracts, Embase, Index Copernicus, EBSCO, African Index Medicus, JournalSeek, Journal Citation Reports/Science Edition, Directory of Open Access Journals (DOAJ), African Journal Online, Bioline International, Open-J-Gate and Pharmacy Abstracts

\section{INTRODUCTION}

Depression is a neurological and psychiatric disease caused by multiple factors. Patients with depression show persistent low mood and cognitive dysfunction [1]. The common manifestations of depression include anorexia, weight loss, inferiority complex, anhedonia, and 
serious suicidal tendency [2]. Currently, depression has become the fourth major disease worldwide.

MicroRNAs (miRNAs), endogenous non-coding small RNAs, have become a hotspot of current research on regulating gene expression. The mechanism underlying miRNA function is related to the formation of a double-stranded untranslated region (UTR) body with 3' complete or incomplete matching form of the target gene, and degrading target gene mRNA or inhibiting transcription and translation, thus affecting various biological processes [3]. Studies have revealed that miRNA expression is dysregulated in peripheral blood of patients with depression [4]. For example, mir-124a, mir-18a, and mir-511 have been found to be upregulated in depression-like rats, and expression of mir-511 was downregulated by antidepressant treatment [5]. Studies have shown that mir-195 is upregulated in the brains of suicidal individuals [6]. However, it is unknown whether mir-195 is associated with depression. Because suicide is one of the manifestations of depression, it has been suggested that mir-195a may be associated with depression.

A growing body of research has shown that the onset of depression is associated with reduced levels of brain-derived neurotrophic factor (BDNF) [7]. BDNF expression was decreased in depressed patients [8], and antidepressant treatment can increase the expression of BDNF and improve depression-like behaviors to a certain extent [9]. BDNF is mainly synthesized by neurons and is most abundant in the cerebral cortex and hippocampus. It is an essential factor involved in the differentiation, development, survival, synaptic plasticity, and regulation of cognitive function of nerve cells [10].

Studies have shown that the number of neurons in depressed mice is significantly reduced, and insufficient or continuous decrease in BDNF content is an important cause of neuronal injury and apoptosis [11]. Antidepressant treatment can enhance the expression of BDNF and CAMP. response element binding protein in the brain [12]. It was found that miRNAs in the central nervous system that bind BDNF mRNA and inhibit BDNF gene translation and protein synthesis include mir-10b, mir-124, mir-134, and mir-155 [13]. Interestingly, TargetScan shows that BDNF appears to be a target of mir-195a. In addition, several articles revealed the interaction between mir-195a and BDNF [14].
Therefore, the present study investigated the effect of the mir-195a-BDNF axis and regulatory mechanism in depression.

\section{EXPERIMENTAL}

\section{Animals}

Male C57BL/6J mice ( $\mathrm{n}=12 ; 8-10$ weeks of age) were housed in a standard Animal Biosafety Level-III Laboratory. Animal care and experimental protocols were carried out following approval by Medical Ethics Committee of Huzhou University (approval no. 20200528-SGY15) and conducted in accordance with the National Institutes of Health Laboratory Animal Care and Use Guidelines [15].

\section{Animal grouping and model establishment}

Chronic social defeat stress (CSDS) was performed as previously described [16]. CSDS was performed between 17:00 and 19:00 before the onset of the dark phase from day 1 to day 10 using a resident-intruder paradigm as previously reported [16].

\section{Social interaction test}

A dual-trial social interaction test was used to investigate avoidance behavior in response to social aversion cues as described previously [16]. In the first 2.5-min trial (target absent), the experimental mice were allowed to freely explore a square open field $(44 \times 44 \mathrm{~cm})$ with a $10 \times 6$ $\mathrm{cm}$ wire cage on one side. During the second 2.5 min trial (target present), the mice were reintroduced to the cage, which now contained an unfamiliar CD1 decommissioned rat.

The video tracking software was used to measure the time in the "interaction zone" (14 $x$ $26 \mathrm{~cm})$. Interaction time was recorded as time that the mice stayed in the interaction zone in the target-present and target-absent conditions.

\section{Sucrose preference test}

The mice were conditioned to drink two bottles of $1 \%$ sucrose solution for $24 \mathrm{~h}$ and one of the bottles of sucrose solution was replaced with water the next day. After $24 \mathrm{~h}$ of fasting and water deprivation, the rats were allowed to drink $1 \%$ sucrose solution $(100 \mathrm{~mL})$ and the same amount of water for $3 \mathrm{~h}$. Sucrose preference (S) was calculated as in Eq 1.

$\mathrm{S}(\%)=\{(\mathrm{Sc} / \mathrm{Wc}+\mathrm{Sc})\} 100$ 
where Sc and Wc are sucrose water consumption and pure water consumption, respectively.

\section{Tail suspension test}

The tail suspension test was carried out as previously described [17]. Mice were suspended on a small metal hook fixed on the top of a box via adhesive tape placed approximately $1 \mathrm{~cm}$ from the tip of the tail. The duration of the immobility period was recorded during the last 5 min of the 6-min test, which reflected the depressive state of the animal.

\section{Cell transfection}

miR-195a inhibitor and mimic used in this experiment were designed and synthesized by Ruibo (Guangzhou, China). Plasmid pcDNA3.1 was purchased from Invitrogen (Carlsbad, CA, USA). HEK-293 cells were seeded in a 6-well plate at a density of $1 \times 10^{4}$ cells $/ \mathrm{mL}$. After $24 \mathrm{~h}$ of culture, the cells were transfected with $50 \mathrm{nM}$ miR-195a mimic and miR-195a inhibitor using Lipofectamine 3000 (Invitrogen \#L3000015). miR-195a and BDNF expression were quantitated by RT-PCR after $24 \mathrm{~h}$ in culture.

\section{Western blotting}

Total protein in the hippocampus and neurons was extracted using total protein extraction kit (Solarbio, Beijing, China) according to the manufacturer's protocol. Equivalent amount of protein was mixed with appropriate loading buffer at $100^{\circ} \mathrm{C}$ for $10 \mathrm{~min}$. Proteins were separated using SDS-PAGE and transferred to PVDF membrane. Membranes were blocked in $5 \%$ skim milk in TBST at room temperature for 1-2 $\mathrm{h}$. Primary antibodies anti-BDNF (1:1000; Cell Signaling Technology, Danvers, MA, USA; \#47808, CST), anti- $\beta$-actin (1:1000; Cell Signaling Technology \#4970) were added and incubated at $4^{\circ} \mathrm{C}$ overnight.

The next day, TBST was removed from the PVDF membranes and anti-rabbit IgG, HRPlinked antibody (1:1000; \#7074, CST) was introduced, then the PVDF membranes were incubated at room temperature for $1 \mathrm{~h}$. Finally, PVDF membranes were washed with TBST. Luminescent solution was added to the gel imager for exposure and photography, and relative expression was calculated using statistical gray value of ImageJ software. $\beta$-actin was used as a reference for loading and at least three independent replicates were performed.

\section{Dual luciferase reporter assay}

To verify the predicted miR-195a binding sequence, the BDNF 3'-UTR fragment containing the predicted binding site or mutant 3'-UTR was cloned into the GV272 vector. After verification by DNA sequencing, the plasmids (with or without miR-195a mimic) were transfected into HEK-293 cells using Lipofectamine 3000 (Invitrogen) according to the manufacturer's instructions. The cells were collected $48 \mathrm{~h}$ after transfection and analyzed using a dual luciferase reporter system.

\section{Surgery and intracranial injection}

Mice were anesthetized with $1 \%$ pentobarbital sodium (50 mg/kg body weight) and then placed in a stereotaxic apparatus and bilaterally implanted with stainless steel guide cannulas in the region of the medial prefrontal cortex (mPFC) (anterior-posterior, $1.9 \mathrm{~mm}$; medial-lateral, 0.5 $\mathrm{mm}$; dorsal-ventral, $-2.3 \mathrm{~mm}$ ). The mice were then individually housed and allowed to recover for at least 7 days. Antagomir-miR-195a/NC (2 $\mu \mathrm{L}, 12 \mathrm{pM}$ ) was microinjected into the mPFC through a cannula.

\section{Statistical analysis}

SPSS 16.0 software was used for statistical analysis of all experimental data. One-way ANOVA was used for comparison among groups. A value of $p<0.05$ was considered statistically significant.

\section{RESULTS}

\section{Expression level of mir-195a was upregulated in CSDS mice}

Mice were successfully rendered depressed using the CSDS model, as shown in Figure $1 \mathrm{~A}$ C. Compared with control mice, CSDS mice expressed less sucrose preference. In addition, when the target was present, CSDS mice showed less social interaction. Finally, CSDS mice show a longer immobility time. These results indicated that the mice in the CSDS group exhibited depression-like behaviors. Consequently, mir-195a expression was upregulated in the peripheral blood of CSDS mice (Figure $1 \mathrm{D}$ ).

\section{Mir-195a targeted BDNF}

In TargetScan (http //www.targetscan.org/ vert 71/), it is assumed that mir-195a binds to the BDNF 3'UTR (Figure $2 \mathrm{~A}$ ). 
A

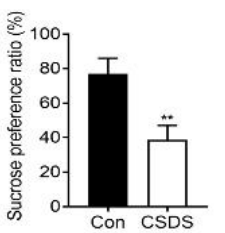

C

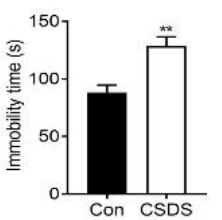

B

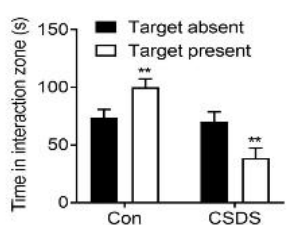

D

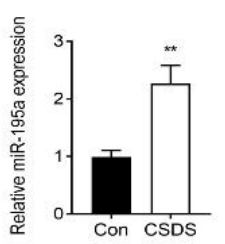

Figure 1: Mir-195a expression was upregulated in CSDS mice. Results of (A) sugar water preference test, (B) social interaction test, and (C) tail suspension test. (D) mir-195a expression was quantitated by quantitative PCR (qPCR). The experiments were repeated three times; ${ }^{* *} p<0.01$, compared with control

HEK-293 cells were transfected with negative control (NC) mimic, mir-195a mimic, NC inhibitor, and mir-195a inhibitor (Figure $2 \mathrm{~B}$ ). Moreover, the relationship between mir-195a and BDNF was validated by dual luciferase reporter assay (Figure 2 C). Mir-195a inhibitor significantly inhibited the expression of mir-195a (Figure 2 D). The qPCR results showed that mir-195a mimic inhibited BDNF mRNA expression level, whereas mir-195a inhibitor increased the level of BDNF mRNA (Figure $2 \mathrm{E}$ ). Similar results were found for protein expression level (Figure $2 \mathrm{~F}$ ). These results suggest that BDNF is a target gene of mir-195a and that it is regulated by mir-195a expression level.

\section{Mir-195a knockdown alleviated depressive symptoms in mice}

To determine the role of mir-195a in depression mice, in vivo experiments were conducted. These experiments showed that mir-195a expression was increased in CSDS mice, and antagomir-mir-195a significantly reduced mir195a expression (Figure 3 A). In addition, antagomir-mir-195a significantly alleviated the depression-like behaviors as characterized by increased sucrose preference (Figure $3 \mathrm{~B}$ ), increased social interaction (Figure $3 \mathrm{C}$ ), and reduced immobility time (Figure 3 D). These observations indicate that mir-195a might contribute to depression and antagomir-mir-195a alleviated depression-like behaviors.

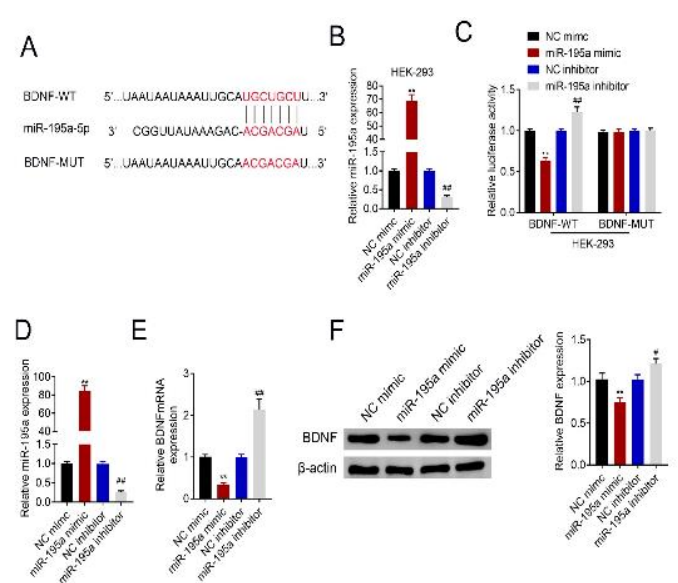

Figure 2: Mir-195a targeted brain-derived neurotrophic factor (BDNF). (A) Target prediction was generated on TargetScan website. (B) Results of qPCR analysis of expression levels of mir-195a and BDNF in HEK-293 cells. (C) Results of luciferase reporter assay to confirm the target relationship between mir-195a and BDNF. (D-E) Results of qPCR analysis to determine the expression of mir-195a and BDNF in primary cortical neurons cells. (F) Results of western analysis (left) and quantitation (right) to determine the expression level of BDNF in primary cortical neurons cells. The experiments were repeated three times; ${ }^{* *} p<0.01$, compared with negative control (NC) mimic; \#\# < 0.01, compared with NC inhibitor
A

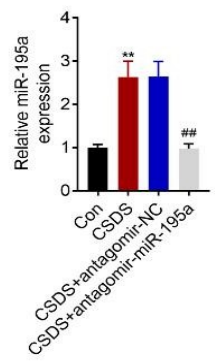

C

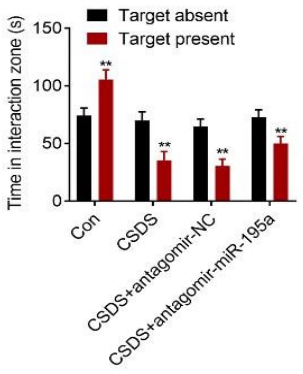

B

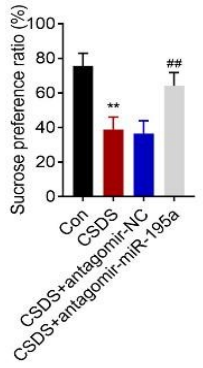

D

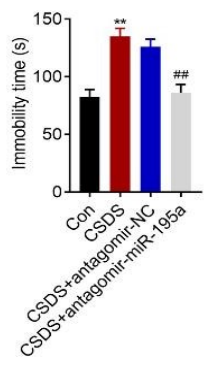

Figure 3: mir-195a knockdown alleviated depressive symptoms in mice. (A) The expression level of mir195a was detected by qPCR. Results of (B) sugar water preference test, (C) social interaction test, and (D) tail suspension test. The experiments were repeated three times; ${ }^{* *} p<0.01$ compared with control; \#\# $p<0.01$, compared with CSDS 


\section{Mir-195a knockdown promoted the expression of BDNF in the hippocampus of mice}

To identify the regulatory mechanism underlying mir-195a function, BDNF expression was determined. As shown in Figure $4 \mathrm{~A}$, expression of BDNF protein was downregulated in CSDS mice, whereas antagomir-mir-195a significantly upregulated BDNF expression. In hippocampal tissue of mice, BDNF expression was downregulated in CSDS, whereas antagomir-mir$195 \mathrm{a}$ restored the baseline expression level (Figure $4 \mathrm{~B}$ ).

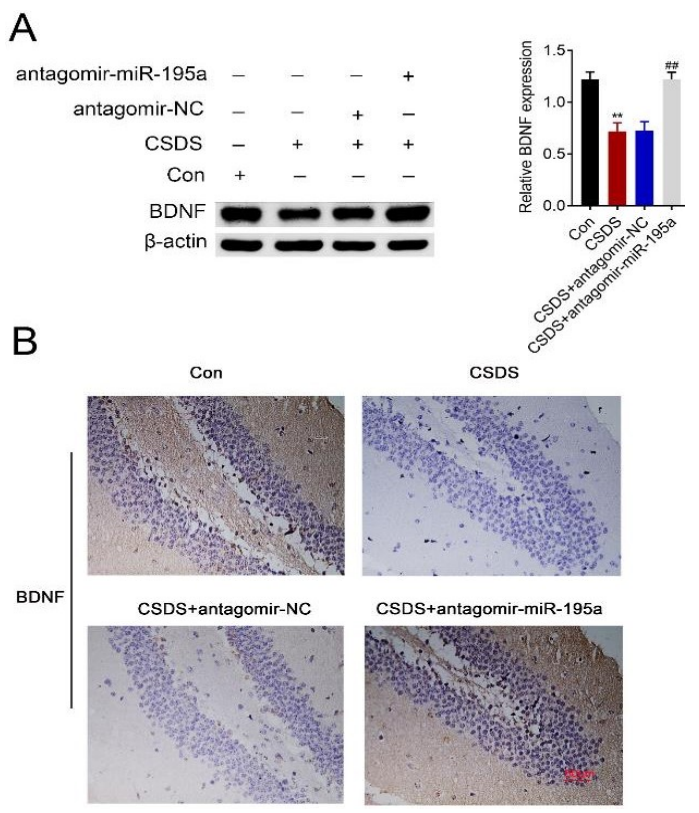

Figure 4: Mir-195a knockdown promoted BDNF expression in the hippocampus of mice. The expression of BDNF was determined by western blotting. (A) Results of western analysis of BDNF expression level (left) and quantitation (right). (B) Immunohistochemical staining of BDNF in hippocampal tissue. Experiments were repeated three times; ${ }^{* *} p<0.01$ compared with control; \#\#p<0.01, compared with CSDS

\section{DISCUSSION}

Depression is often accompanied by cognitive or behavioral changes or disorders. Changes in cognitive ability and behavior have been reported in depressed rats, possibly due to damage to the limbic system, of which the hippocampus is an important component [5]. The establishment of animal models of central nervous disease is one of the most commonly used research methods [12]. The CSDS model is one of the most widely used depression models at present [17]. This study used the CSDS model to construct a mouse depression model. In recent years, depression and antidepressant treatment have been associated with adult hippocampal neurogenesis [17].

A neuron is the basic unit of a nerve. In vitro and in vivo experiments have demonstrated that miR195a regulates cell damage in the hippocampus of depressed mice by down-regulating BDNF. BDNF has been reported to influence a variety of complex physiological processes, including learning and memory, which are also essential for the survival of neural stem cells, thus regulating neurite growth and maturation during brain development [10]. In this study, the data showed that expression of BDNF mRNA and protein was downregulated in hippocampal tissues and neurons of the depression model mice, suggesting that downregulation of BDNF plays a key role in depression in mice.

Regulation of transcription and translation is a complex multi-factor process. miRNA achieves negative feedback regulation by shearing mRNA and inhibiting its translation [18]. miRNAs are important regulators of normal development and physiology, and many miRNAs serve as biomarkers for various diseases. Abnormal miRNA expression in depressed patients has been reported to be associated with stress responses and antidepressant medication [18].

In this paper, bioinformatics-based prediction and luciferase assay proved that BDNF is the target gene of miR-195a. miR-195a has a variety of biological functions based on the roles of its target genes. For example, miR-195a is closely related to various cancers [19]. Recent studies have shown that miR-195a is involved in the pathogenesis of depression and/or suicide [6]. The results of this study showed that expression of BDNF mRNA and protein was downregulated in the depression model mice, whereas miR195a expression was upregulated. In combination with the results of behavioral tests, miR-195a and BDNF are thought to regulate depression-like behaviors in mice.

To confirm that miR-195a regulates BDNF expression, miR-195a antagomir was used in the study. miR-195a antagomir interferes with the expression of miR-195a. The results derived from this study demonstrated that miR-195a knockdown via miR-195a inhibitor/antagomir removed the inhibition of BDNF by miR-195, thus increasing BDNF expression. This further demonstrated that BDNF is the target of miR195a, which regulates BDNF expression. The results of this study suggest that the high expression level of miR-195a was positively correlated with depression, and inhibition of miR- 
195 a expression might be helpful for the treatment of depression.

\section{CONCLUSION}

This study suggests that the mechanism of depression in mice may involve up-regulation of miR-195a expression in hippocampal tissues and neurons, leading to the downregulation of BDNF mRNA and protein expression. miR-195a, as a regulator of BDNF, may be a target for the prevention and treatment of depression.

\section{DECLARATIONS}

\section{Conflict of interest}

No conflict of interest is associated with this work.

\section{Contribution of authors}

We declare that this work was done by the authors named in this article and all liabilities pertaining to claims relating to the content of this article will be borne by the authors. Xuping Wen and Mingshuan Lin designed the study, supervised the data collection, analyzed and interpreted the data, prepared the manuscript for publication, and reviewed the draft of the manuscript. All authors have read and approved the manuscript.

\section{Open Access}

This is an Open Access article that uses a funding model which does not charge readers or their institutions for access and distributed under the terms of the Creative Commons Attribution License (http://creativecommons.org/licenses/by/ 4.0) and the Budapest Open Access Initiative (http://www.budapestopenaccessinitiative.org/rea d), which permit unrestricted use, distribution, and reproduction in any medium, provided the original work is properly credited.

\section{REFERENCES}

1. Jie $Y$, Shuang $D$, Xinjie Z. Effects of concurrent treatment with amitriptyline hydrochloride tablets and fluoxetine hydrochloride on therapeutic indicator levels in patients with depression. Trop J Pharm Res 2019; 18(2): 403408.

2. Alexopoulos GS, Sirey JA, Banerjee $S$, Kiosses DN, Pollari C, Novitch RS, Artis A, Raue PJ. Two behavioral interventions for patients with major depression and severe COPD. Am J Geriat Psychiat 2016; 24(11): 964974.
3. Saliminejad K, Khorram Khorshid HR, Soleymani Fard S, Ghaffari SH. An overview of microRNAs: Biology, functions, therapeutics, and analysis methods. J Cell Physiol 2019; 234(5): 5451-5465.

4. Babenko O, Kovalchuk I, Metz GA. Stress-induced perinatal and transgenerational epigenetic programming of brain development and mental health. Neurosci Biobehav Rev 2015; 48: 70-91.

5. $X u$ J, Wang R, Liu $Y$, Wang $W$, Liu D, Jiang $H$, Pan $F$. Short- and long-term alterations of FKBP5-GR and specific microRNAs in the prefrontal cortex and hippocampus of male rats induced by adolescent stress contribute to depression susceptibility. Psychoneuroendocrinology 2019; 101: 204-215.

6. Lopez JP, Fiori LM, Gross JA, Labonte B, Yerko V, Mechawar N, Turecki G. Regulatory role of miRNAs in polyamine gene expression in the prefrontal cortex of depressed suicide completers. Int J Neuropsychoph 2014; 17(1): 23-32.

7. Kumar A, Dogra S, Sona C, Umrao D, Rashid M, Singh SK, Wahajuddin M, Yadav PN. Chronic histamine 3 receptor antagonism alleviates depression like conditions in mice via modulation of brain-derived neurotrophic factor and hypothalamus-pituitary adrenal axis. Psychoneuroendocrinology 2019; 101: 128-137.

8. Nunes PV, Nascimento CF, Kim HK, Andreazza AC, Brentani HP, Suemoto CK, Leite REP, de Lucena Ferretti-Rebustini RE, Pasqualucci CA, Nitrini R. Low brain-derived neurotrophic factor levels in post-mortem brains of older adults with depression and dementia in a large clinicopathological sample. J Affect Disorders 2018; 241: 176-181.

9. Wilkinson ST, Kiselycznyk C, Banasr M, Webler RD, Haile C, Mathew SJ. Serum and plasma brain-derived neurotrophic factor and response in a randomized controlled trial of riluzole for treatment resistant depression. J Affective Disorders 2018; 241: 514-518.

10. McAllister AK, Katz LC, Lo DC. Neurotrophins and synaptic plasticity. Annu Rev Neurosci 1999; 22(1): 295318.

11. Wang $Y$, Jia A, Ma W. Dexmedetomidine attenuates the toxicity of $\beta$-amyloid on neurons and astrocytes by increasing $B D N F$ production under the regulation of HDAC2 and HDAC5. Mol Med Rep 2019; 19(1): 533540.

12. Cao C, Xiao J, Liu M, Ge Z, Huang R, Qi M, Zhu H, Zhu $Y$, Duan J-a. Active components, derived from Kai-xinsan, a herbal formula, increase the expressions of neurotrophic factor NGF and BDNF on mouse astrocyte primary cultures via CAMP-dependent signaling pathway. J Ethnopharmacol 2018; 224: 554-562.

13. Shen J, $X u$ L, Qu C, Sun $H$, Zhang J. Resveratrol prevents cognitive deficits induced by chronic unpredictable mild stress: Sirt1/miR-134 signalling pathway regulates CREB/BDNF expression in hippocampus in vivo and in vitro. Behav Brain Res 2018; 349: 1-7.

Trop J Pharm Res, December 2020; 19(12): 2542 
14. Ehinger $Y$, Phamluong $K$, Darevesky D, Welman $M$, Moffat JJ, Sakhai SA, Whiteley EL, Berger AL, Laguesse S, Farokhnia $M$ et al. Differential correlation of serum BDNF and microRNA content in rats with rapid or late onset of heavy alcohol use. Addict Biol: 2020: e12890.

15. Health Nlo: Guide for the care and use of laboratory animals: National Academies; 1985.

16. Deng Z-F, Zheng H-L, Chen J-G, Luo Y, Xu J-F, Zhao G, Lu J-J, Li H-H, Gao S-Q, Zhang D-Z. miR-214-3p targets $\beta$-catenin to regulate depressive-like behaviors induced by chronic social defeat stress in mice. Cerebral Cortex 2019; 29(4): 1509-1519.

17. Menard C, Pfau ML, Hodes GE, Kana V, Wang VX, Bouchard S, Takahashi A, Flanigan ME, Aleyasin H,
LeClair KB et al: Social stress induces neurovascular pathology promoting depression. 2017, Nat Neurosci, 20(12):1752-1760.

18. Williams AE, Moschos SA, Perry MM, Barnes PJ, Lindsay MA. Maternally imprinted microRNAs are differentially expressed during mouse and human lung development. Dev Dyn 2007; 236(2): 572-580.

19. Pushpavalli S, Ramaiah MJ, Srinivas C, Mukhopadhya D, Aditya JL, Kumbhare RM, Bhadra U, Bhadra MP. Effect of Benzothiazole based conjugates in causing apoptosis by Regulating p53, PTEN and MAP Kinase proteins affecting miR-195a and miR-101-1. Cancer Cell Int 2011; 11: 36 